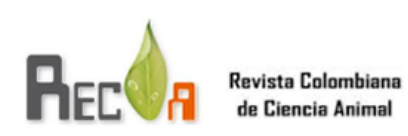

Revista Colombiana de Ciencia Animal ISSN: 2027-4297

reciaeditor@unisucre.edu

Universidad de Sucre

Colombia

\title{
Caracterização da dieta de Melanosuchus niger (Spix, 1825) e Caiman crocodilus (Linnaeus, 1758) na Resex Lago do Cuniã, Rondônia
}

da Costa, Igor David; Neto dos Santos Nunes, Natalia

Caracterização da dieta de Melanosuchus niger (Spix, 1825) e Caiman crocodilus (Linnaeus, 1758) na

Resex Lago do Cuniã, Rondônia

Revista Colombiana de Ciencia Animal, vol. 11, núm. 1, 2019

Universidad de Sucre, Colombia

DOI: https://doi.org/10.24188/recia.v11.n1.2019.708

Los autores permiten a RECIA reimprimir el material publicado en él. En caso de que un autor quiera traducir o usar una publicación parcial o completa de nuestro Diario, el autor debe obtener un permiso por escrito del editor de la revista.

Esta obra está bajo una Licencia Creative Commons Atribución 4.0 Internacional. 


\section{Caracterização da dieta de Melanosuchus niger (Spix, 1825) e Caiman crocodilus (Linnaeus, 1758) na Resex Lago do Cuniã, Rondônia}

Characterization of the diet of Melanosuchus niger (Spix, 1825) and Caiman crocodilus (Linnaeus, 1758) in the Resex Lago do Cuniã, Rondônia

Igor David da Costa

DOI: https://doi.org/10.24188/recia.v11.n1.2019.708

Universidade Federal Fluminense, Instituto do Noroeste

Fluminense de Educação Superior, Avenida João Jasbick, s/

$n^{\circ}$ - Bairro: Aeroporto, CEP: 28470-000, Santo Antônio

de Pádua, Rio de Janeiro, Brasil. Universidade Federal de

Recepção: 19 Fevereiro 2019

Rondônia, Mestrado Profissional em Gestão e Regulação

de Recursos Hidricos (PROF-AGUA), Campus Ji-Paraná,

Aprovação: 25 Março 2019

Rua Rio Amazonas, 351 - Jardim dos Migrantes, CEP:

76900-726, Ji-Paraná, Rondônia, Brasil, Brasil

igorbiologia@yahoo.com.br

iD http://orcid.org/0000-0002-8556-5507

Natalia Neto dos Santos Nunes

Universidade Federal de Rondônia, Programa de Pós-

Graduação em Ciências Ambientais, Av. Norte Sul 7300,

CEP: 76.940-000, Rolim de Moura, Rondônia, Brasil.,

Brasil

nataliansnunes@gmail.com

(iD http://orcid.org/0000-0001-5744-1358

\section{ReSUMO:}

O objetivo deste estudo foi caracterizar a dieta de indivíduos machos de Melanosuchus niger e Caiman crocodilus em um lago da Reserva Extrativista do Lago do Cuniã, Rondônia. Foram coletados intestinos dos animais em novembro/2016, estação chuvosa, fornecidos pela Cooperativa local (COOPCuniã). As cavidades abdominais dos jacarés foram abertas e seu estômago foi retirado e armazenado em álcool a 70\%, e os alimentos foram analisados. Foram analisados 82 estômagos, 42 de M. niger e 40 de C. crocodilus. Alimentos como fragmentos de aves, anfíbios, crustáceos, insetos e material vegetal foram ingeridos pelas espécies estudadas. No entanto, os itens mais representativos na dieta de $M$. niger e $C$. crocodilus foram peixes da ordem Characifomes e Siluriformes sem importância comercial.

Palavras-Chave: Alimentação, Amazônia, Conservação, Crocodilianos, Ecologia trófica.

\section{Abstract:}

The aim of this study was to characterize the diet of male individuals of Melanosuchus niger and Caiman crocodilus in a lake at the Reserva Extrativista do Lago do Cuniã, Rondônia. We collected guts of the animals in November/2016, rainy season, provided by the local Cooperative (COOPCuniã). Caimans abdominal cavities were opened and their stomach were removed, after the gut contents were stored in $70 \%$ alcohol, and food items were analyzed. We analyzed 82 stomachs, 42 of $M$. niger and 40 of $C$. crocodilus. Food items such as fragments of birds, amphibians, crustaceans, insects, and plant material were ingered by the species studied. However, the most representative items in the diet of M. niger and C. crocodilus were fishes from the Characifomes and Siluriformes order of no commercial importance.

KeYwords: Amazonia, Conservation, Crocodilians, Feeding, Trophic ecology. 


\section{INTRODUÇÃO}

Atualmente são reconhecidos 8 gêneros e 23 espécies viventes de crocodilianos que são divididas nas Famílias Crocodylidae, Alligatoridae e Gavialidae, pertencentes à subordem Eusuchia (1). As cinco espécies de crocodilianos que ocorrem no Brasil pertencem à Família Alligatoridae, popularmente chamadas de jacarés (2), Caiman crocodilus, Melanosuchus niger, Paleosuchus trigonatus, Paleosuchus palpebrosus e Caiman latirostris. Destas, apenas C. latirostris não ocorre na Amazônia (1).

A espécie M.niger vulgarmente conhecida como jacaré-açu, apresentam focinho largo, liso e com uma notável crista pré-ocular, coloração dorsal negra com listras verticais brancas ou amareladas, e na mandíbula manchas acinzentadas nos jovens e marrom nos adultos (3), podem alcançar $6 \mathrm{~m}$ de comprimento total (4), apresentam tamanho médio da ninhada de 34 ovos, variando entre 23 e 48 (5) e as populações naturais sofrem impactos de ações antrópicas, tais como a criação de represas, o desmatamento e a caça (6). Já o jacaretinga, C. crocodilus, é caracterizada por uma crista ou testa em crescente localizado logo acima dos olhos e sobre o dorso do focinho, apresentam uma crota ossificada em frente dos olhos, os juvenis são amarelos com manchas pretas nos lados do corpo e cauda, já os adultos, modificam sua coloração para verde-oliva escuro (3). Podem alcançar 2,5m de comprimento total (7) e o número médio de ovos encontrado é de 25 , variando de 14 a 38 (8). Os impactos sobre a espécie são os mesmos relatados para $M$. niger.

Crocodilianos predam grande variedade de invertebrados e vertebrados $(2,3)$, e sua estratégia oportunista de alimentação pode afetar seu comportamento, condição corporal, crescimento e reprodução (4). Como observado nos estudos de Barão-Nóbrega et al (3), que concluíram que fêmeas de C. crocodilus, em período de nidificação, ou seja, períodos duradouros de privação alimentar, consomem presas disponíveis nas proximidades do ninho. $\mathrm{Na}$ maioria das espécies é observado um incremento de até $70 \%$ no consumo de peixes, e um maior consumo de presas maiores, à medida que os indivíduos aumentam de tamanho (5).

As espécies Melanosuchus niger (Spix, 1825) e Caiman crocodilus (Linnaeus, 1758) habitam simpatricamente o lago Cuniã, Rondônia. Embora a dieta e o comportamento alimentar de ambas as espécies tenha sido investigada $(2,4,9,10,11,12)$, ainda são poucas as informações sobre a dieta destas espécies no Estado de Rondônia e em áreas protegidas na Amazônia, principalmente em uma RESEX que apresenta como principal recurso explorado as espécies $M$. niger e $C$. crocodilus. Nesse sentido, o presente estudo teve por objetivo avaliar a composição da dieta de C. crocodilus e M. niger na Reserva Extrativista do Lago Cuniã (Resex Cuniã), Rondônia, Brasil.

\section{MATERIAIS E MÉTODOS}

A Resex Cuniã (Figura 1) localiza-se a cerca de $130 \mathrm{~km}$ do município de Porto Velho. A estação chuvosa ocorre de novembro a abril e a estação seca de maio a outubro (13). 

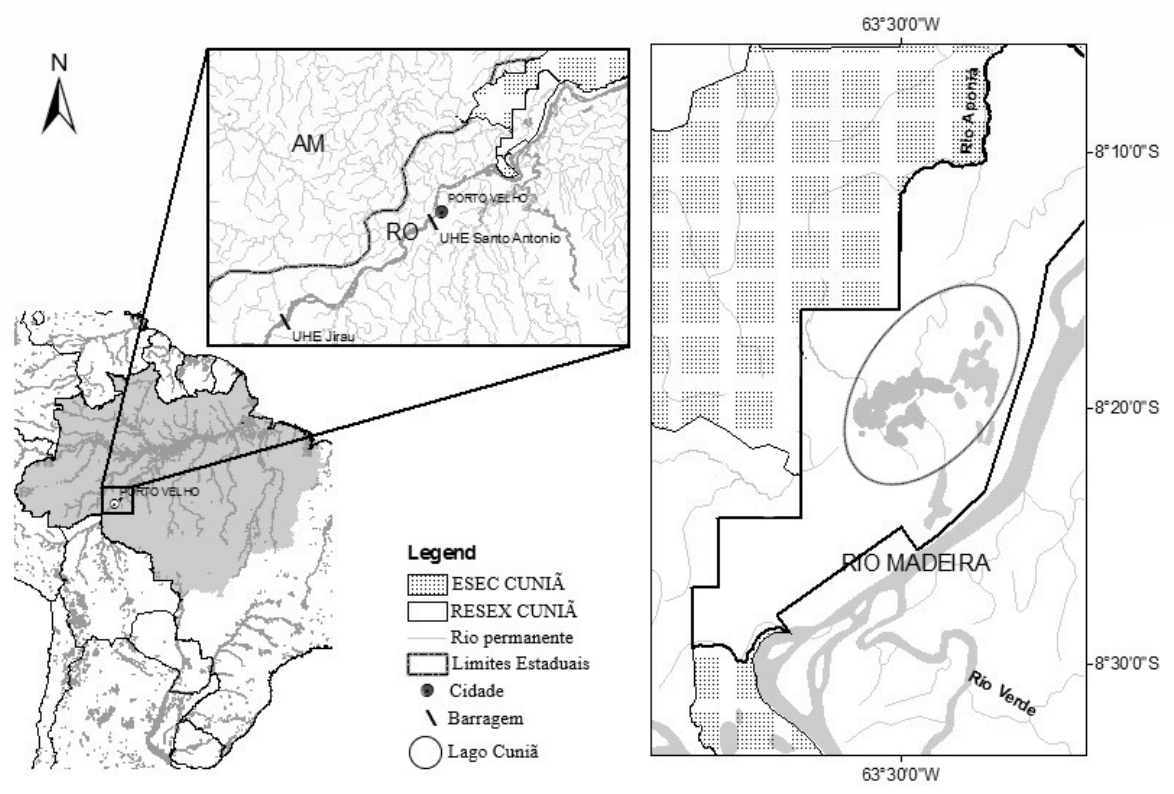

FIGURA 1

Área de estudo com indicação do Lago Cuniã, Rondônia, Brasil.

O Lago Cuniã é uma importante área protegida, localizada na Reserva Extrativista do Lago do Cuniã (RESEX Cuniã), que apresenta mais de 140 espécies de peixes $(6,7)$, sustenta pescarias locais sujeitas a um plano de gestão ambiental e outras atividades de uso sustentável dos recursos naturais $(6,14)$ como a captura e abate de jacarés. Com uma área superficial de 18.000 ha o Lago Cuniã esta situado na parte inferior do rio Madeira, e compreende um complexo de aproximadamente 60 lagos e canais, que são interconectados durante o período de enchente do rio, abrigando grandes áreas de macrófitas aquáticas, liteira submersa, troncos marginais e sendo um dos poucos lagos de várzea de grande porte com águas turvas (7), promovendo uma grande complexidade estrutural, que contribui para a grande biodiversidade animal na área.

O Lago Cuniã apresenta a população de jacarés com o número de machos superior ao número de fêmeas (15). A Cooperativa da Resex (COOPCuniã) foi fundada em abril de 2011 no intuito de comercializar os produtos da RESEX (16). A Resex Cuniã produz farinha, açaí, castanha-do-brasil e explora o manejo de algumas espécies de peixes e duas espécies de jacarés, $C$. crocodilus e $M$. niger (16). A cooperativa é gerenciada por um grupo de Gestores que são responsáveis pelas atividades dentro e fora da RESEX, mas o objetivo de todas as atividades da cooperativa é garantir o cumprimento de sua missão: "realizar o manejo dos recursos com foco na sustentabilidade" (16). O modelo de produção seguido na RESEX é do tipo harvesting, autorizado em 2011 pelo IBAMA com finalidade de controle populacional.

Os jacarés machos de ambas as espécies foram capturados no lago da Resex Cuniã, em novembro de 2016, pelos membros da COOPCuniã. As capturas foram realizadas à noite, usando laço (enforcador) de cabo de aço. Após a captura, os animais foram contidos em curral redondo, foram tomadas as medidas biométricas de comprimento total $(\mathrm{m})$ com auxílio de uma trena e massa total $(\mathrm{kg})$ com uma balança de 0,1 g de precisão e peso máximo de $100.000 \mathrm{~g}$ e higienizados. Posteriormente os indivíduos foram abatidos com pistola pneumática na área interna do frigorífico, eviscerados e os estômagos foram fornecidos para análise. As amostragens foram autorizadas pelo Instituto Chico Mendes de Conservação da Biodiversidade (ICMBio, Licença: 53594-1/2017).

Os estômagos foram armazenados em alcool a 70\% e os itens alimentares foram analisados e identificados ao menor nível taxonômico possivel. A frequência de ocorrência (\%Freq.) e a frequência volumétrica (\%Vol.) foram utilizadas para quantificar o conteúdo dos estômagos (17). A \%Freq. foi calculada pelo número de 
estômagos que continham cada item alimentar, calculando a porcentagem em relação ao total de estômagos. Para o \%Vol., o volume de cada item foi obtido usando a porcentagem em relação ao valor total de cada conteúdo do tubo digestivo. $\mathrm{O}$ volume foi mensurado utilizando uma proveta graduada, onde o volume foi medido em mililitro (18). Esses dados foram combinados no Índice de importância alimentar (IAi) proposto por Kawakami \& Vazzoler (19) e adaptado por Hahn et al (20), de acordo com a equação: $\mathrm{IAi}=\left(\mathrm{Fi}{ }^{*} \mathrm{Vi}\right)$ / $\left.\Sigma\left(\mathrm{Fi}^{*} \mathrm{Vi}\right)\right)^{*} 100$, onde $\mathrm{i}=1,2, \ldots \mathrm{n}$ de itens alimentares; $\mathrm{Fi}=$ frequência de ocorrência de um determinado item alimentar; $\mathrm{Vi}=$ volume de um determinado item alimentar. Foi calculada a frequência de ocorrência dos itens alimentares ingeridos pelas espécies, sendo estes classificadas segundo a escala de Dajoz (21) como: constantes, quando o item está presente em mais de $50 \%$ das amostras; acessórias, entre 25 e $50 \%$ e acidentais, em menos de $25 \%$ das amostras. Nesse caso cada amostra representou um estômago.

\section{RESULTADOS}

Foram analisados um total de 82 estômagos, 42 de $M$. niger e 40 estômagos de $C$. crocodilus. O comprimento e massa média de $M$. niger foram de $1,8 \pm 0,1 \mathrm{~m}$ e $17,0 \pm 5,5 \mathrm{Kg}$ e de $C$. crocodilus foram de 1,7 $\pm 0,1 \mathrm{~m}$; $19,0 \pm 5,0 \mathrm{Kg}$, respectivamente. Ambas as espécies ingeriram um total de 14 itens alimentares, variando em itens de origem animal, como fragmentos de aves, fragmentos de peixes, anfíbios, crustáceos e insetos, e itens de origem vegetal, como Brachiaria sp (Figura 2 e 3). Os itens Hoplosternum littorale (IAi $=0,55$ ), seguido de Brachiaria sp. (IAi $=0,20)$ e penas ( $\mathrm{IAi}=0,11)$ foram os mais ingeridos por C.crocodilus, enquanto para M. niger os itens mais importantes foram Brachiaria sp. (IAi =0,39), Characiformes sp $1(\mathrm{IAi}=0,19)$ e Loricariidae (IAi $=0,17$; Tabela 1; Figura 4). 
TABELA 1

Frequência relativa (\% Freq.), Volume (\% Vol.) e Índice de importância alimentar (IAi) de C. crocodilus e $M$. niger na Resex Lago do Cuniã, coletados em novembro de 2016. Con $=$ constantes, Ace $=$ acessórias e Aci $=$ acidentais (19).

\begin{tabular}{|c|c|c|c|c|c|c|}
\hline \multirow{2}{*}{ Itens } & \multicolumn{3}{|c|}{ C. crocodilus } & \multicolumn{3}{|c|}{ M. niger } \\
\hline & \% Freq. & $\%$ Vol. & IAi & \% Freq. & $\%$ Vol. & IAi \\
\hline Vegetal & & & & & & \\
\hline Brachiaria sp. & 0,35 & 0,06 & $0,2081^{\text {Ace }}$ & 14,31 & 29,22 & $0,3939^{\mathrm{ACi}}$ \\
\hline $\begin{array}{l}\text { Animal } \\
\text { Insecta }\end{array}$ & & & & & & \\
\hline Coleoptera & 0,11 & 0 & $0,0051^{A C i}$ & 7,1 & 0,21 & $0,0013^{\mathrm{Aci}}$ \\
\hline Belostomatidae & 0,05 & 0 & $0,0005^{\mathrm{Aci}}$ & - & - & - \\
\hline Crustacea & & & & & & \\
\hline Caranguejo & 0,05 & 0,01 & $0,0080^{\mathrm{ACi}}$ & - & - & - \\
\hline $\begin{array}{l}\text { Anphibia } \\
\text { Anuro } \\
\text { Peixes }\end{array}$ & - & - & - & 7,1 & 12,1 & $0,0815^{\mathrm{ACl}}$ \\
\hline Hoplosternum littorale & 0,11 & 0,5 & $0,5503^{\mathrm{Aci}}$ & 7,18 & 11,1 & $0,0753^{\mathrm{Aci}}$ \\
\hline Loricariidae & - & - & - & 14,3 & 13 & $0,1756^{\mathrm{ACI}}$ \\
\hline Ossos de Siluriformes & 0,05 & 0,01 & $0,0824^{\mathrm{ACl}}$ & 21,43 & 3,01 & $0,0602^{\mathrm{Aci}}$ \\
\hline Characiforme sp 1 & 0,05 & 0,06 & $0,0314^{\mathrm{ACl}}$ & 7,1 & 28,8 & $0,1944^{\mathrm{Aci}}$ \\
\hline Characiforme sp 2 & - & - & - & 7,1 & 2,01 & $0,0138^{\mathrm{ACi}}$ \\
\hline Ossos de Characiformes & - & - & - & 7,1 & 0,6 & $0,0038^{\mathrm{Aci}}$ \\
\hline $\begin{array}{l}\text { Nadadeiras de } \\
\text { Characiformes } \\
\text { Aves }\end{array}$ & 0,05 & 0,11 & $0,0613^{\mathrm{Aci}}$ & - & - & - \\
\hline Ossos de aves & 0,05 & 0,01 & $0,0062^{\mathrm{ACl}}$ & - & - & - \\
\hline Penas & 0,05 & 0,2 & $0,1104^{\mathrm{Aci}}$ & 7,1 & 0,11 & $0,0003^{\mathrm{Aci}}$ \\
\hline
\end{tabular}

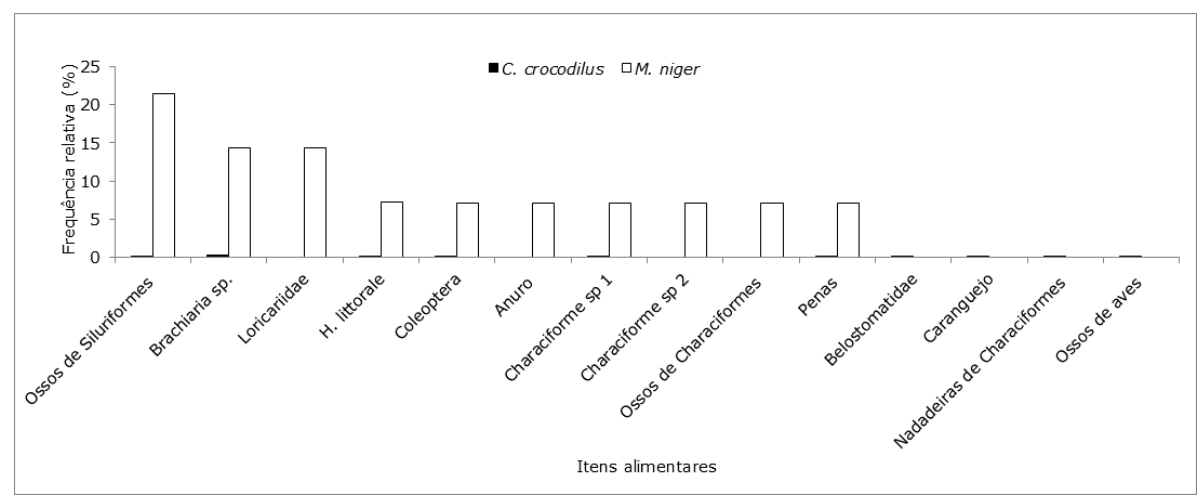

FIGURA 2

Percentual de frequência relativa dos itens alimentares consumidos por $C$. crocodilus e M. niger na Resex Lago do Cuniã, coletados em novembro de 2016 
Igor David da Costa, et al. Caracterização da dieta de Melanosuchus niger (Spix, 1825) e Caiman cr...

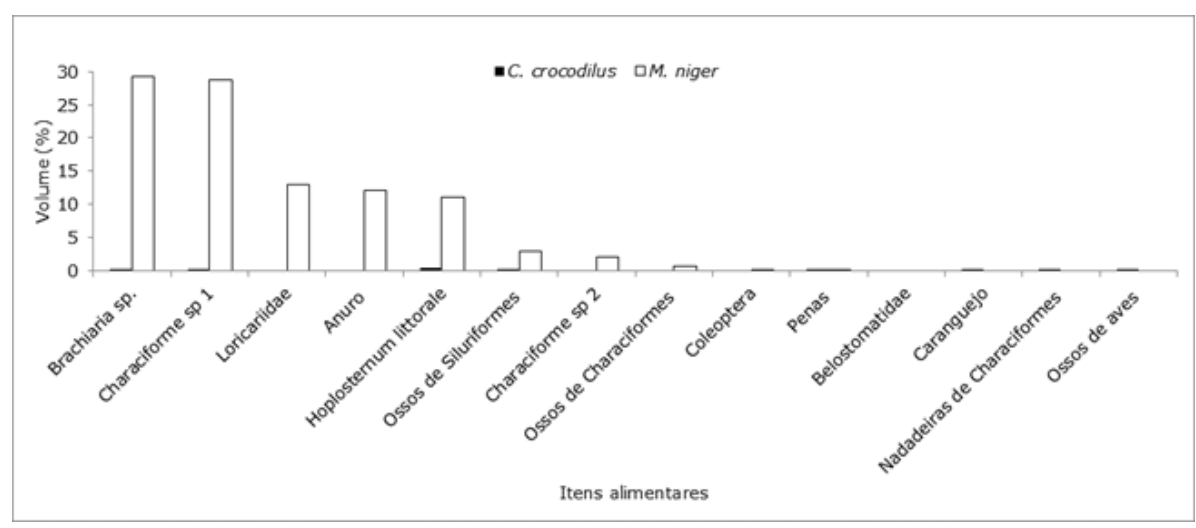

FIGURA 3

Percentual de volume dos itens alimentares consumidos por C. crocodilus e M. niger na Resex Lago do Cuniã, coletados em novembro de 2016. 


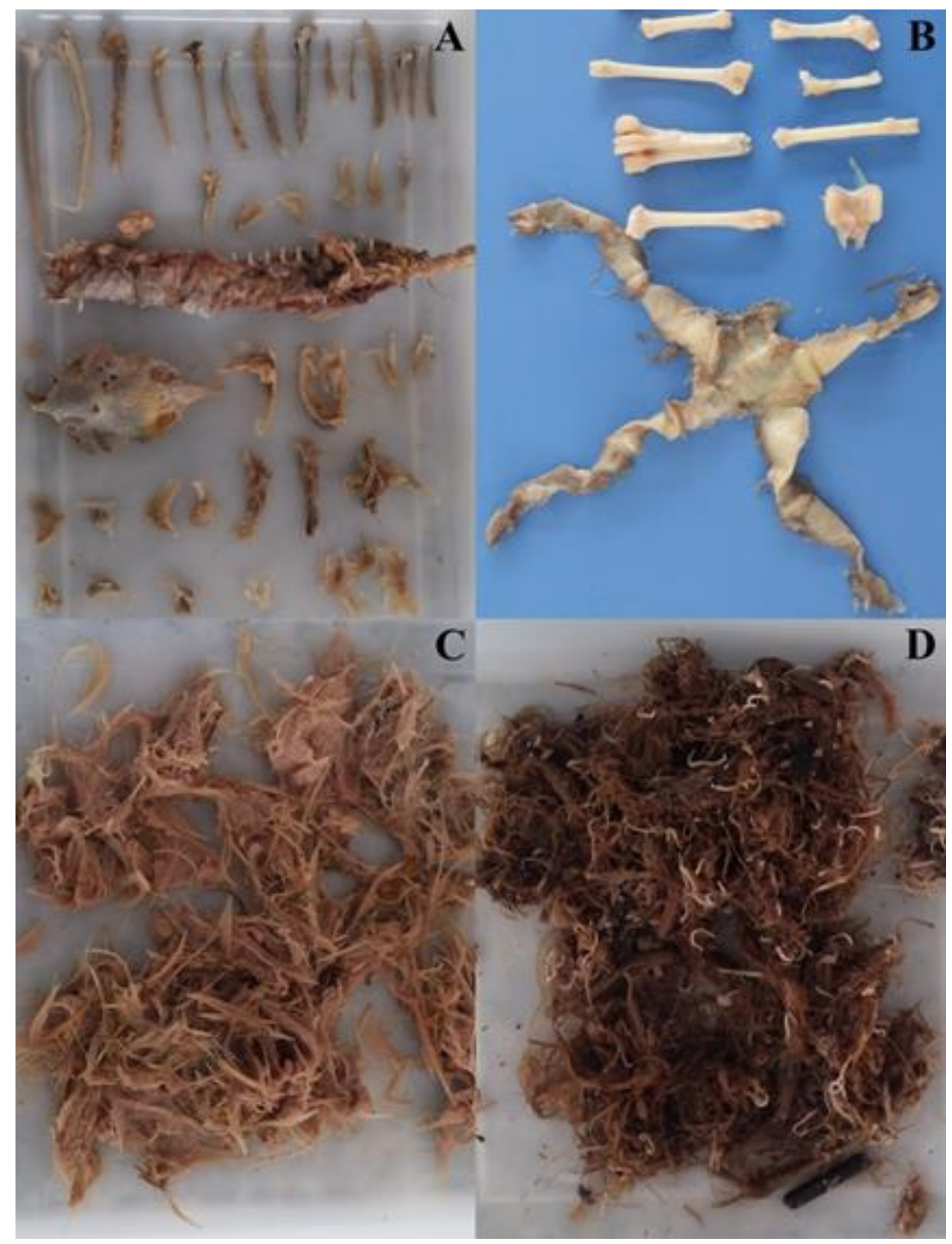

FIGURA 4

Itens alimentares da dieta de $M$. niger e $C$. crocodilus na Resex Lago do Cuniã, Rondônia. A: esqueleto de peixe da Ordem Siluriformes. B: ossos e epiderme de anuro. C: penas. D: Brachiaria sp. com presença de nematoides.

As espécies de jacarés estudadas consumiram uma variedade de itens alimentares, todavia os itens mais representativos em volume, frequência e número de espécies relacionam aos peixes. Espécies do grupo dos Characifomes, Perciformes, Siluriformes, Beloniformes, Symbranchiformes e Osteoglossiformes também foram encontrados em conteúdos estomacais de $C$. crocodilus (22) e $M$. niger (23), no Pantanal e bacia amazônica, respectivamente.

\section{DISCUSSÃO}

Os peixes foram o item mais consumido por ambas as espécies estudadas no lago Cuniã. As espécies $C$. crocodilus e $M$. niger ingeriram um total de 14 itens alimentares, todavia $C$. crocodilus consumiu uma maior variedade de itens invertebrados enquanto $M$. niger ingeriu mais itens do grupo dos vertebrados. Os crocodilianos são predadores generalistas, se alimentam de uma variedade de presas animais (23) e sua dieta pode variar de acordo com as espécies, habitat, disponibilidade de presas e condições ambientais, como 
temperatura e níveis de água (24). As espécies $M$. niger e $C$. crocodilus se alimentam principalmente de insetos (coleópteros), aranhas, caranguejos, moluscos e vertebrados como peixes, aves, répteis e mamíferos $(9,11,23,25)$.

Aproximadamente $82 \%$ dos itens alimentares registrados na dieta de $M$. niger e C. crocodilos neste estudo, são os mesmos registrados para estas espécies na Amazônia $(9,11,23,26)$ e nos Llanos Venezuelanos (26,27). A alta incidência de peixes na dieta das espécies está de acordo com informações anteriores $(23,26)$, e pode estar relacionado ao fato de que os animais analisados são machos, considerando que estes utilizam com maior frequência o habitat aquático, quando comparado às fêmeas, que no período reprodutivo passam por longos períodos de incubação dos ovos em terra firme (28). Estudos de Da Silveira e Magnusson (23), no arquipélago de Anavilhanas, Amazonas, apontam para uma maior proporção de peixes consumidos por $C$. crocodilus em lagos da região, sendo tal resultado provavelmente relacionado à maior disponibilidade de peixes, menor profundidade e correnteza neste ambiente (23), que aumenta a vulnerabilidade das presas aos crocodilianos, quando comparado a ambientes lóticos, que apresentam maior profundidade e correnteza.

A espécie C. crocodilus ocupa poças com vegetação flutuante e herbáceas aquáticas marginais, sendo estes habitats permanentes e não dependentes diretamente do pulso de inundação (29). Estudos de Da Silveira \& Magnusson (23) apontam a ingestão de material vegetal pelas espécies estudadas, sendo esta considerada como acidental pelos autores, todavia, no presente estudo o item Brachiaria sp. foi classificado como acessório na dieta de $C$. crocodilus. Considerando que a proteína vegetal não é digerida ou assimilada por crocodilianos (30), a elevada importância de material vegetal na dieta das espécies poderia estar associada ao microhabitat utilizado por espécies de peixes. Macrófitas aquáticas são utilizadas por insetos, aves e peixes como berçário, refúgio e sítio de forrageamento (31). A migração entre habitat de macrófitas por peixes dulcícolas têm sido estudada, sendo visto que a dinâmica de movimentação entre macrófitas e águas abertas por Characiformes, e outras ordens de peixes, é relacionada a tácticas anti-predação (refúgio) e forregeamento (31). Dessa forma, é sugerido com o estudo que a presença de material vegetal no estômago de ambas as espécies, esta mais associado a captura de peixes e outros organismos, que utilizam tal microhabitat, do que o consumo deste item propriamente dito.

Segundo Santos et al (22), em estudos no Pantanal brasileiro, C. crocodilus consumiu principalmente animais aquáticos, abundantes na fauna terrestre (mamíferos e aves). No entanto, em um estudo em Caño Negro, Costa Rica, a espécie C. crocodilusfuscus ingeriu frequentemente aves aquáticas (32). Mamíferos são encontrados com frequência na dieta de $C$. crocodilus $(27,10)$, dado não corroborado neste estudo. $\mathrm{O}$ consumo de organismos terrestres pode estar associado a variação na disponibilidade de peixe no ambiente ao longo do ano, em decorrência da flutuação hidrológica no ambiente estudado (25).

As proteínas animais contêm mais aminoácidos essenciais do que as proteínas vegetais. As proteínas de alto valor nutritivo são encontradas na carne, peixes e aves (33). Entretanto, os peixes são a grande fonte de ácidos graxos, devido à sua alimentação fitoplanctônica e/ou zooplanctônica (34). Crocodilianos em habitat natural, que têm sua dieta composta por peixes, moluscos, crustáceos, e espécies silvestres, possuem normalmente valores maiores de ácidos graxos poliinsaturados do que saturados (9).

Pesquisas preliminares sugeriram que $M$. niger pode afetar ecologicamente $C$. crocodilus (35). Todavia, apesar de ambas as espécies utilizarem itens alimentares similares em suas dietas, a pesquisa não pode indicar a existência de competição trófica, considerando que é necessário avaliar a complexidade estrutural, presença de microhabitats do Lago Cuniã e disponibilidade de presas para as espécies analisadas. Destacamos o maior consumo de peixes pelas espécies de jacarés estudadas, dessa forma, medidas de manejo e conservação da ictiofauna regional são importantes a fim de manter a sobrevivência e prevalência de $M$. niger e $C$. crocodilus e promover outras fontes de renda para as comunidades residentes na Resex Cuniã. 


\section{REFERÊNCIAS}

1. Vasconcelos WR. Diversidade genética e estrutura populacional dos crocodilianos jacaré-açú (Melanosuchus niger) e jacaré-tinga (Caiman crocodilus) da Amazônia. [Tesis M.Sc.]. Manaus Brasil: Universidade Federal do Amazonas 2005. URL disponível em: https://bdtd.inpa.gov.br/bitstream/tede/1920/5/William\%20Rangel \%20Vasconcelos.pdf

2. Magnusson WE. Mortality of eggs of the crocodile Crocodylus porosus in northern Australia. Journal of Herpetology. 1982; 1(1):121-130. DOI: http://dx.doi.org/10.2307/1563804

3. Pereira AC, Malvasio A. Síntese das características da ordem Crocodylia, fatores de influência em estudos populacionais e aspectos de seleção e uso de habitat para Caiman crocodilus e melanosuchus niger no estado do Tocantins, Brasil. Biota Amazônia. 2014; 4(1): 111-118. DOI: http://dx.doi.org/10.18561/2179-5746/ biotaamazonia.v4n1p111-118

4. Medem F. Los Crocodylia de Sur América. 1ª edição. Bogotá, Colombia: Colciencias; 1983.

5. Mendonça S, Coutinho M. Relatório técnico sobre as atividades desenvolvidas na Reserva Extrativista do Lago do Cuniã e Estação Ecológica de Cuniã, Porto Velho, Rondônia. 1 1a edição. São Paulo, Brasil: Centro Nacional de Pesquisa e Conservação de Répteis e Anfíbios/RAN/ ICMBio; 2010. URL disponível em: http://www.icmbio.gov.br/ran/images/stories/publicacoes/relatorios/ relat\%C3\%B3rio_cunia_resultados2004a2008.pdf

6. Da Silveira R, Thorbjarnarson JB. Conservation implications of commercial hunting of black and spectacled caiman in the Mamirauá Sustainable Development Reserve, Brazil. Biological Conservation. 1999; 88(1):103-109.DOI: https://doi.org/10.1016/S0006-3207(98)00084-6

7. Ross JP. Win a few, lose a few. Crocodile Specialist Group Newsletter. Melbourne. 1998; 17(1)2-3.

8. Campos ZDS. Efeito do habitat na fecundidade das fêmeas, sobrevivência e razão sexual dos jovens de jacarés-do-pantanal. 1a edição. Campo Grande, Brasil: Embrapa Pantanal; 2003. URL disponível em:https:// www.infoteca.cnptia.embrapa.br/bitstream/doc/809826/1/BP42.pdf

9. Magnusson WE, Da Silva EV, LIMA AP. Diets of Amazonian crocodilians. Journal of Herpetology. 1987; 21(2):85-95. DOI: http://dx.doi.org/10.2307/1564468

10. Barão-Nóbrega JAL, Marioni B, Dutra-Araújo D, Botero-Arias R, Nogueira AJ, Magnusson WE. Nest attendance influences the diet of nesting female spectacled caiman (Caiman crocodilus) in central Amazonia, Brazil. The Herpetological Journal 2016; 26(2):65-71. URL: https://www.researchgate.net/profile/Diogo_Dutra-Araujo2/ publication/283073843_Nest_attendance_influences_the_diet_of_nesting_female_spectacled_caiman_Caiman_crocodilu links/5757453f08ae04a1b6b6910a.pdf

11. Rice AN. Diet and condition of American alligators, Alligator mississippiensis, in three central Florida lakes [Tesis M.Sc.]. Florida: University of Florida; 2004 URL disponível em: http://ufdcimages.uflib.ufl.edu/UF/ E0/00/43/67/00001/rice_a.pdf

12. Hossain MS, Jaman MF, Ahmed M, Rahman MM, Uddin M. Food consumption of saltwater crocodile (Crocodylus Porosus) in a reptile farm of Bangladesh. Bangladesh J Zool. 2013; 41(2):173-179.

13. Queiroz LJ, Torrente-Vilara G, Vieira FG, Ohara WM, Zuanon J Doria CRC. Fishes of Cuniã Lake, Madeira River Basin, Brazil. Check list 2013; 9(3):540-548. DOI: http://dx.doi.org/10.15560/9.3.540

14. Vieira FG, Matsuzaki AA, Barros BSF, Ohara WM, Paixão AC, Torrente-Vilara G, Zuanon J, Doria CRC. Catálogo de Peixes da Estação Ecológica de Cuniã. $1^{a}$ edição. Porto Velho: Edufro; 2016. URL: https:// ppbio.inpa.gov.br/sites/default/files/PeixesdaEsecCunia_0.pdf

15. Mendonça S, Coutinho M. Bases biológicas para o manejo do jacaré-açu (Melanosuchus niger) na Reserva Extrativista do Lago do Cuniã, Rondônia. 1a edição. Goiás: CBH; 2009.

16. ICMBIO. RESEX Lago do Cuniã inicia atividades de produção do projeto de manejo de jacarés. [Internet]. 1st ed. Brasilia: ICMBIO; 2011. [citado 23 março de 2019]. URL 
Disponível em: http://www.icmbio.gov.br/portal/comunicacao/noticias/20-geral/2034-resex-do-lago-docunia-inicia-atividades-de-producao-do-projeto-de-manejo-dejacares.html?highlight=WyJjdW5pYSJd

17. Hyslop EJ. Stomach contents analysis - a review of methods and their application. Journal of Fish Biology 1980; 17(4):411-429. DOI: http://dx.doi.org/10.1111/j.1095-8649.1980.tb02775.x

18. Hellawell JM, Abel R. A rapid volumetric method for the analysis of the food of fishes. Journal of Fish Biology. 1971;3(1):29-37. DOI: https://doi.org/10.1111/j.1095-8649.1971.tb05903.x

19. Kawakami E, Vazzoler G. Método gráfico e estimativa de índice alimentar aplicado no estudo de alimentação de peixes. Bobn Inst Oceanogr. S. Paulo. 1980; 29(2):205-207. URL: http://www.scielo.br/pdf/bioce/v29n2/ v29n2a43.pdf

20. Hahn NS, Agostinho AA, Goitein R. Feeding ecology of curvina Plagioscion squamosissimus (Heckel, 1840) (Osteichthyes, Perciformes) in the Itaipu reservoir and Porto Rico floodplain. Acta Limnologica Brasiliensia 1997, 9(1):11-22. URL: http://ablimno.org.br/acta/pdf/acta_limnologica_contents9E_files/Art.02\%20Vol \%20IX.pdf

21. Dajoz R. Princípios de ecologia. 1a edição. Rio de Janeiro: Editora Artmed; 1996.

22. Santos SA, Nogueira MS, Pinheiro MS, Campos Z, Magnusson WE, Mourao GM. Diets of Caiman crocodilus yacare from different habitats in Brazilian Pantanal. Herpetological Journal. 1996; 1(6):111-117. URL: https://www.thebhs.org/publications/the-herpetological-journal/volume-6-number-4october-1996/1460-02-diets-of-caiman-crocodilus-yacare-from-different-habitats-in-the-brazilian-pantantal/ file

23. Da Silveira R, Magnusson WE. Diets of spectacled and black caiman in the Anavilhanas Archipelago, Central Amazonia, Brazil. Journal of Herpetology 1999, 33(2):181-192. DOI: http://dx.doi.org/10.2307/1565713

24. Richardson KC, Webb GJW, Manolis SC. Crocodiles: Inside out. A guide to the crocodilians and their functional morphology. $2^{a}$ edição. Australia: Surrey Beatty and Sons; 2002.

25. Marioni B, Da Silveira R, Magnusson WE, Thorbjarnarson J. Feeding behavior of two sympatric caiman species, Melanosuchus niger and Caiman crocodilus, in the Brazilian Amazon. Journal of Herpetology 2008; 42(4):768-772. URL: https://www.jstor.org/stable/40060575

26. Somaweera R, Brien ML, Platt SG, Manolis C, Webber BL. Direct and indirect interactions with vegetation shape crocodylian ecology at multiple scales. Freshwater Biology 2019; 64(2):257-268. DOI: http:// dx.doi.org/10.1111/fwb.132

27. Thorbjarnarson JB. Diet of the spectacled caiman (Caiman crocodilus) in the central Venezuelan llanos. Herpetologica 1993; 49(1):108-117. URL: https://www.jstor.org/stable/3892691

28. Cunha FAG, Barboza RSL, Rebêlo GH. Communal Nesting of Caiman crocodilus (Linnaeus, 1758)(Crocodylia: Alligatoridae) in Amazon Forest Floodplain, Brazil. Herpetology Notes. 2016; 9(1):141-144. URL: https:// www.biotaxa.org/hn/article/viewFile/8858/20835

29. Moldowan PD, Laverty TM, Emmans CJ, Stanley RC. Diet, Gastric Parasitism, and Injuries of Caimans (Caiman, Melanosuchus, and Paleosuchus) in the Peruvian Amazon. South American J of Herpetology 2016; 11(3):176-183.DOI: http://dx.doi.org/10.2994/SAJH-D-16-00012.1

30. Pinheiro MS, Lavorenti A. Growth of broad-nosed caiman, Caiman latirostris (Daudin, 1802) hatchlings, fed with diets of animal origin. Braz J Biol. 2001; 61(3):421-429. DOI: http://dx.doi.org/10.1590/ S1519-69842001000300011

31. Magalhães ERS, Yamamoto KC, Anjos HDB, Loebens SC, Soares MGM. Bancos de macrófitas aquáticas em lago de várzea: alimentação de duas espécies de peixes na região de Manaus, Amazonas, Brasil. Acta of Fisheries and Aquatic Resources 2015; 3(1):25-40. DOI http://dx.doi.org/10.2312/ActaFish.2015.3.1.25-40

32. Escobedo-Galván AH. Population structure and sex ratio in Caiman crocodilus in Caño Negro, Costa Rica. Iheringia. Série Zoologia 2008; 98(4):489-492. DOI: http://dx.doi.org/10.1590/S0073-47212008000400012

33. Tonial IB, Bravo CEC, de Souza NE, Matsushita M, Furuya WM, Visentainer JV. Nutritional quality of lipids tilapia (Oreochromis niloticus) fed with supplemented diets with soybean oil. Alimentos e Nutricao (Brazilian Journal of Food and Nutrition) 2011; 22(1):103-113. 
34. Sinclair AJ, O'dea K. Fats in human diets through history: Is the Western diet out of step? In; Wood JD, Fisher AV, editores. Reducing fat in meat animals. Vol 1, 1 ed. London: Elsevier Applied Science; 1990.

35. Campos Z, Muniz F, Magnusson WE. Where are the black caimans, Melanosuchus niger (Crocodylia: Alligatoridae), in the Xingu River basin, Brazil? Herpetology Notes 2018; 11(1):1021-1024. DOI: https:// www.biotaxa.org/hn/article/viewFile/38826/36566

Los autores permiten a RECIA reimprimir el material publicado en él. En caso de que un autor quiera traducir o usar una publicación parcial o completa de nuestro Diario, el autor debe obtener un permiso por escrito del editor de la revista.

CC BY 